\title{
MANUTENÇÃO EM PÁ CARREGADEIRA: UM ESTUDO DE CASO PARA REDUÇÃO DAS HORAS PARADAS *
}

Gabriel Mori da Rocha ${ }^{1}$ Luciano José Vieira Franco ${ }^{2}$ Jussara Fernandes Leit ${ }^{2}$ Heleno Alves Barbosa ${ }^{2}$ Anderson Correa Lopes ${ }^{2}$ Ernandes José Correia ${ }^{2}$

\section{Resumo}

Nas grandes empresas, existe a preocupação com o número de horas paradas dos equipamentos para realizar manutenção, pois os custos de suas intervenções tornaram-se um fator determinante nos ganhos de uma organização. Neste contexto, esta pesquisa tem como objetivo reduzir o número de horas paradas não programadas, em manutenção da pá carregadeira. $O$ estudo abordou as lubrificações manuais e automáticas. Os dados foram coletados por meio de observações, documentos internos e entrevistas com os mecânicos. Desta forma, foi verificado o histórico das paradas não programadas da carregadeira no período entre 01/11/2015 e 30/04/2016, e identificou-se que a lubrificação manual era responsável por 25,8\%, podendo chegar a 32,8\% devido à geração de demais problemas no equipamento. A partir disso, foi proposta e implementada a lubrificação automática. Como resultado, a pesquisa identificou que houve redução das horas de paradas não programadas na carregadeira, diminuição dos custos com relação aos insumos e maior disponibilidade do equipamento.

Palavras-chave: Paradas não programadas, Lubrificação, Carregadeira.

\section{MAINTENANCE IN SPARE PARTS: A CASE STUDY TO REDUCE STOPED TIME}

\begin{abstract}
In large companies, there is concern about the number of hours of equipment stopped to perform maintenance, because the costs of their interventions have become a determining factor in the return of an organization. In this context, this research has the objective to reduce the number of unscheduled downtime in maintenance of the wheel loader. The study addressed manual and automatic lubrications. Data were collected through observations, internal documents and interviews. In this way, the history of unscheduled stops of the loader was verified in the period from 11/11/2015 to 04/30/2016, and it was identified that the manual lubrication was responsible for $25.8 \%$ and could reach $32.8 \%$ due to the generation of other problems in the equipment. From this, automatic lubrication was proposed and implemented. As a result, the research identified that there was a reduction in the number of unplanned downtime on the loader, lower costs with respect to inputs and greater equipment availability.
\end{abstract}

Keywords: Unscheduled stops, Lubrication, Loader Machine.

1 Graduando em Engenharia de Produção, Fundação Presidente Antônio Carlos, de Barão de Cocais, Minas Gerais, Brasil.

2 Engenheiro, Professor da Fundação Presidente Antônio Carlos, Conselheiro Lafaiete, Minas Gerais, Brasil. 


\section{INTRODUÇÃO}

A globalização da economia e a competitividade no mercado fizeram com que as empresas observassem melhor o seu processo produtivo. Com isso, a manutenção dos equipamentos passou a ser um dos pilares para as organizações atingirem os objetivos.

Em verdade, nas grandes empresas, é nítida a preocupação com a qualidade das manutenções, que é considerada aquela que coloca os equipamentos em perfeito estado de operações, com o maior índice de disponibilidade e menor custo possível. Em qualquer organização, para que todas as máquinas funcionem de maneira adequada, é necessário que elas estejam em boas condições e confiabilidade para operação. E isso, é proporcionado pelo setor de manutenção ao desenvolverem os diversos tipos de manutenções: corretivas, preventivas e preditivas.

Para identificar qual o melhor método de realizar a manutenção, as organizações devem realizar estudos sobre as falhas que ocorrem nos equipamentos, isto para conseguir adequar o processo. Um processo eficiente tem foco nos pontos onde as falhas ocorrem. Logo, por meio de uma análise detalhada, os responsáveis têm como saná-las.

Um tipo de manutenção realizada na maioria dos equipamentos industriais são as lubrificações, visto que praticamente todas as máquinas industriais precisam desta para funcionar corretamente. Em realidade, os custos com a falta da lubrificação são altos, assim a atenção neste processo pode evitar gastos com a manutenção dos equipamentos.

Segundo Collins ${ }^{1}$ o lubrificante é parte essencial no processo de lubrificação, e é considerado como uma substância que visa separar as superfícies deslizantes, reduzir o atrito e o desgaste, remover o calor gerado por atrito, acentuar a operação suave e fornecer uma vida operacional aceitável a elementos de máquinas deslizantes.

Collins $(2006)^{1}$ e Ipiranga $(2016)^{2}$ relatam que a necessidade de lubrificação se explica quando duas superfícies metálicas deslizam uma em relação à outra. A este movimento existe uma resistência, que é denominada "atrito". O atrito é totalmente indesejável no caso das partes metálicas em movimento de um equipamento, pois implica em geração excessiva de calor e desgaste das peças, além da perda de energia pelo contato entre elas, causando uma perda de rendimento e consumo adicional de combustível.

Pizzani (2016) ${ }^{3}$ acrescenta que quando recobertos por um lubrificante, os pontos de atrito das superfícies sólidas fazem com que o atrito sólido seja substituído pelo atrito fluido, ou seja, sem atrito entre uma superfície sólida e um fluido. Nessas condições, o desgaste entre as superfícies é reduzido. Além dessa redução do atrito, outros objetivos são alcançados com a lubrificação, se a substância lubrificante for selecionada corretamente, obtendo-se: menor dissipação de energia na forma de calor; redução da corrosão; redução de vibrações e ruídos; redução do desgaste.

Santos (2016) ${ }^{4}$ informa que com a evolução dos lubrificantes, esses passaram a acumular novas funções, como proteção contra a corrosão, auxílio à vedação, transferência de calor e retirada de produtos indesejáveis do sistema. Os lubrificantes podem ser gasosos como o ar; líquidos como os óleos em geral; semisólidos como as graxas e sólidos como a grafita, o talco, a mica, e outros.

Neste trabalho, foi abordada a lubrificação em pá carregadeira que trabalha na extração de minério de ferro em uma empresa da Região do Médio Piracicaba em Minas Gerais. O estudo foi baseado nas lubrificações manuais e automáticas, com o 
objetivo de reduzir o número de horas paradas desse equipamento em manutenção para realizar lubrificação.

Nesse contexto, Franzen (2005) ${ }^{5}$ afirma que a lubrificação manual diária é de difícil execução, devido à más condições climáticas; necessidade de produção, não podendo parar o maquinário; aspectos de segurança; logística - tal como disponibilidade do comboio e a localização do equipamento -; muitos pinos da caçamba e da lança necessitam ser lubrificados em mais de uma posição para distribuir o lubrificante uniformemente e proteger componentes sob carga pesada; características da mão de obra, pois nem todos os profissionais executam a lubrificação adequadamente.

O mesmo autor afirma também que a lubrificação manual não consegue espalhar a graxa dentro do mancal e nem retirar completamente a graxa velha. A falha na lubrificação de cada ponto, em cada máquina, a cada dia, afeta sua programação, seus custos de manutenção e seu desempenho nos prazos contratados.

Quando feita de forma inadequada, a lubrificação manual gera vários custos, tais como gastos aumentados com peças e reparos; perda de produção com lubrificação manual e reparos imprevistos; contaminação desgastando pinos, buchas e outros componentes; queda do valor de revenda para equipamentos usados; desperdício de lubrificante devido à lubrificação excessiva.

Lubrificação automatizada, também denominada de lubrificação centralizada, Eximport $(2015)^{6}$ esclarece que é uma forma de aplicação de lubrificante em todos os pontos de uma máquina (ou de um conjunto de máquinas simultaneamente), nas quantidades, pressões e frequências corretas, a partir de um reservatório central. $O$ lubrificante é conduzido limpo, sem manuseio nem desperdício e com a máquina em operação a partir de um reservatório central e com a máquina operando.

Eximport $(2015)^{6}$ ressalta que os sistemas de lubrificação centralizada são classificados conforme o seu princípio de operação. Os diversos sistemas são empregados praticamente em qualquer tipo de máquina ou equipamento, sejam móveis ou estacionários, desde pequenas máquinas têxteis com alguns pontos de lubrificação, até grandes instalações siderúrgicas com milhares de pontos. Os principais resultados obtidos com uso destes sistemas são: eficiência, segurança e economia.

De acordo com a Carmassa Brasil $(\mathrm{CMB}, 2016)^{7}$, com a aplicação da lubrificação centralizada em partes como engrenagens, rolamentos, barramentos, fusos, as chances de um dano ou quebra emergencial diminuem drasticamente, afinal, sua vida útil aumenta cerca de 5 vezes devido ao sistema de lubrificação centralizada.

$\mathrm{Na}$ visão da fabricante de rolamentos SKF $(2016)^{8}$, normalmente o sistema de lubrificação centralizada injeta pequenas quantidades medidas de lubrificante em intervalos frequentes, enquanto as máquinas estão em funcionamento. O fluxo de graxa criado pela bomba é dividido por dispositivos de medição progressiva e distribuído a cada rolamento conforme suas necessidades. Esses sistemas foram desenvolvidos para proporcionar um método relativamente simples e barato de automatizar a lubrificação de rolamentos, pinos e buchas das máquinas.

Franzen $(2005)^{5}$ apresenta um sistema centralizado com operação automática, chamado de QuickLub, que consiste em uma bomba elétrica com injetores de graxa, distribuidores para direcionar e regular a quantidade de graxas, mangueiras e tubulações que conduzem a graxa aos mancais. A Figura 1 mostra o sistema de uma Bomba automática QuickLub. 


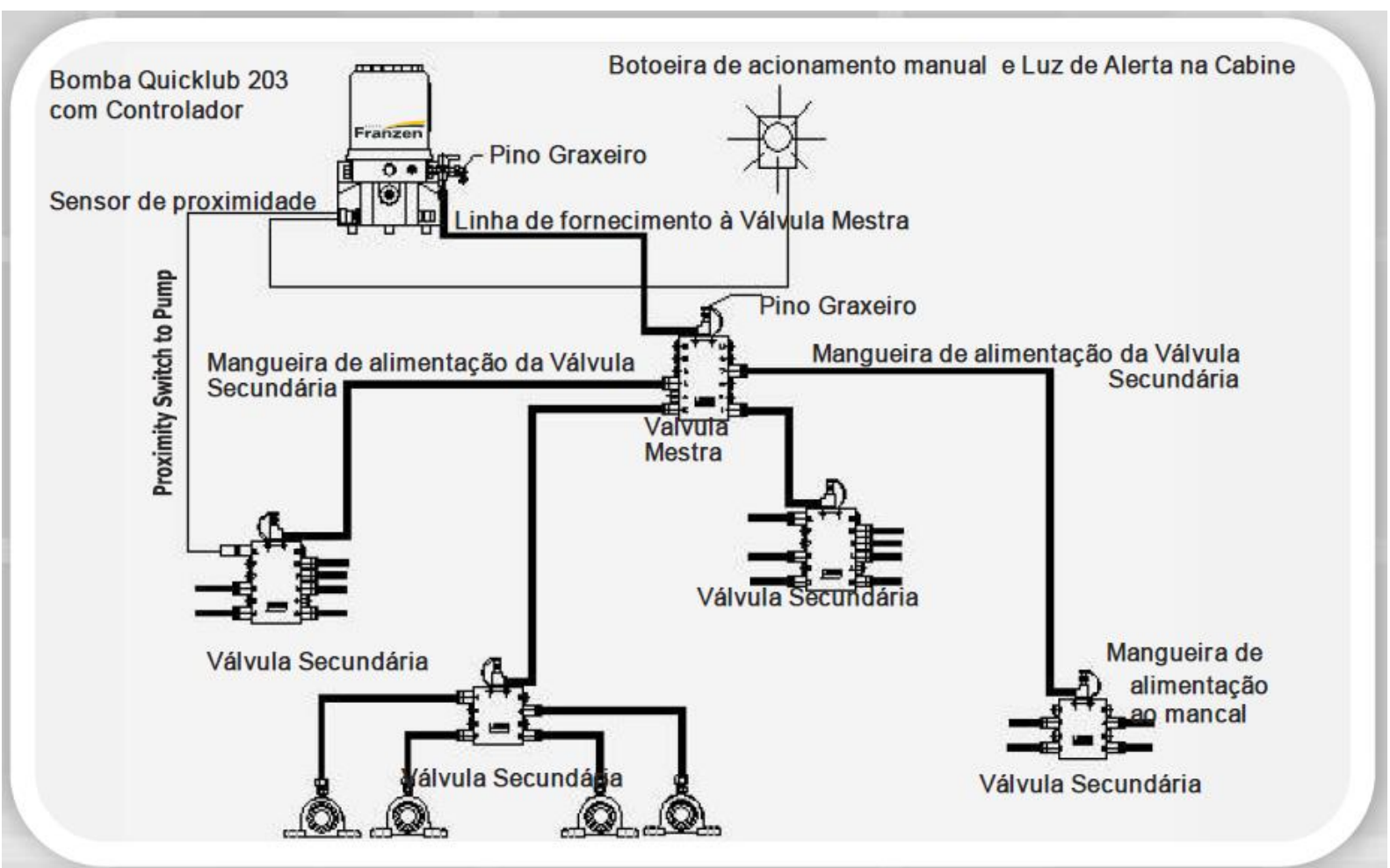

Figura 1 - Esquema Básico de Lubrificação Centralizada (Franzen, 2005)

Conforme Figura 1, o sistema contém uma linha de distribuição de graxa principal e outras linhas de distribuição de graxa secundárias, distribuindo a graxa de acordo com a necessidade de cada ponto.

Franzen (2005) ${ }^{5}$ informa que todo o sistema funciona automaticamente por meio de um temporizador ligado à bomba elétrica que injeta a graxa para lubrificar os mancais em ciclos de tempo pré-determinados, possuindo como benefícios:

- Evitar a parada do equipamento para lubrificação dos mancais, aumentando a produtividade do equipamento;

- Ganho de minutos/dia no aumento da produtividade por máquina ao lubrificar em operação;

- Reduz o custo com mão de obra em comparação com o sistema tradicional;

- Prolonga a vida útil dos mancais do equipamento;

- Reduz o custo com graxas, pois o sistema automático é programado para mandar somente a quantidade de graxa necessária para cada ponto sem perdas para o ambiente;

- Reduz a contaminação do solo por excesso de graxa nos mancais;

- Melhora a segurança ao eliminar a prática diária do trabalhador ao subir nas máquinas para lubrificar;

- Aumenta o valor de revenda do equipamento.

Como foi expresso anteriormente, os equipamentos necessitam realizar lubrificações para se manter em perfeito estado de conservação e funcionamento. Isso também é aplicado às pás mecânicas, também conhecidas como carregadeiras.

A revista Manutenção e Tecnologia (M\&T, 2010) ${ }^{9}$ informam que as carregadeiras são equipamentos articulados, isto é, são constituídos por dois módulos. O módulo do implemento (dianteiro) é formado pela caçamba, braço de elevação e articulações, componentes hidráulicos de acionamento e uma estrutura de eixo para montagem desses sistemas e suporte da carga. $O$ módulo de potência 
(traseiro) contém o motor, conversor de torque, transmissão, eixo traseiro, e componentes hidráulicos, fornecendo potência para escavação, remoção e elevação de material, e para tração dos módulos dianteiros.

Para melhor entendimento, Umarizal Terraplanagem e Locação (ULT, 2016) explicam que as carregadeiras são máquinas de terraplenagem que realizam escavação, mas como o próprio nome sugere, são especializadas no carregamento de caminhões basculantes, seja em obras de terraplenagem para remoção de terra, ou em mineração carregando minérios em geral.

\section{MATERIAIS E MÉTODOS}

Esta pesquisa é um estudo de caso, realizada em uma empresa de mineração de minério de ferro da Região do Médio Piracicaba em Minas Gerais, no período de agosto a novembro de 2016. Estudo caracterizado como exploratório, explicativo e documental, baseado no processo de extração de minério de ferro com pá carregadeira, e tem como objetivo reduzir o número de horas paradas não programadas em manutenção.

Os dados possuem natureza qualitativa e quantitativa e foram coletados por meio de observações, documentos e entrevistas.

No desenvolvimento da pesquisa, as observações tiveram o propósito de verificar as paradas não programadas e buscar melhorias no processo de lubrificação.

Os documentos referem-se aos relatórios gerenciais do setor de manutenção. Por meio desses dados de tempo e de paradas não programadas para manutenção, foram coletados as horas paradas e seus motivos. Esses foram utilizados para analisar a disponibilidade dos equipamentos e a incidência de quebras. Assim, procedeu-se a análise do histórico de paradas não programadas da carregadeira e realizou-se um estudo para identificar as soluções para reduzir o número de horas paradas para lubrificação.

Posterior à modificação do sistema de lubrificação da carregadeira, foram analisados os dados de tempo de paradas não programadas com a finalidade de demonstrar o resultado da nova tecnologia utilizada.

Por fim, uma entrevista com os cinco mecânicos que executam lubrificação nas carregadeiras foi realizada. A entrevista teve o objetivo de identificar a satisfação dos empregados com o novo método de lubrificação e as possíveis melhorias para a realização de suas atividades.

\section{RESULTADOS E DISCUSSÃO}

Neste trabalho, inicialmente, foram coletados os dados de paradas não programadas da carregadeira no período de 01/11/2015 a 30/04/2016. Os valores podem ser verificados na Tabela 1, apresentada a seguir. 
Tabela 1 - Tempo de paradas não programadas da carregadeira (motivo).

\begin{tabular}{l|c|c}
\hline \multicolumn{1}{c|}{ Motivo } & $\begin{array}{c}\text { Tempo de parada } \\
\text { em Horas }\end{array}$ & Data \\
\hline Buzina não funciona & 4 & $06 / 11 / 2015$ \\
\hline Caçamba rangendo & 5 & $23 / 11 / 2015$ \\
\hline Ar condicionado não está resfriando & 7 & $15 / 12 / 2015$ \\
\hline Vazamento hidráulico & 6 & $19 / 12 / 2015$ \\
\hline Caçamba rangendo & 4,5 & $02 / 01 / 2016$ \\
\hline Folga nos pinos da caçamba & 7 & $23 / 01 / 2016$ \\
\hline Quebra do eixo cardam & 9 & $04 / 02 / 2016$ \\
\hline Caçamba rangendo & 4 & $16 / 02 / 2016$ \\
\hline Buzina não funciona & 5 & $21 / 02 / 2016$ \\
\hline Pino de lubrificação obstruído & 3,5 & $27 / 02 / 2016$ \\
\hline Baixo rendimento & 11 & $05 / 03 / 2016$ \\
\hline Vazamento hidráulico & 4,5 & $09 / 03 / 2016$ \\
\hline Caçamba rangendo & 5,5 & $23 / 03 / 2016$ \\
\hline Farol queimado & 4 & $01 / 04 / 2016$ \\
\hline Superaquecimento do motor & 10 & $12 / 04 / 2016$ \\
\hline Ar condicionado não está resfriando & 6 & $16 / 04 / 2016$ \\
\hline Pino de lubrificação obstruído & 4,5 & $23 / 04 / 2016$ \\
\hline Freio de estacionamento não funciona & 4 & $27 / 04 / 2016$ \\
\hline
\end{tabular}

Conforme Tabela 1, verifica-se que vários são os motivos das paradas não programadas das carregadeiras. A partir desses dados, foi calculado o tempo de horas paradas das carregadeiras conforme sistema do equipamento. Os valores e 0 percentual em relação ao total de horas paradas, segundo sistema, podem ser observados na Tabela 2.

Tabela 2 - Horas paradas da carregadeira para manutenção conforme sistema.

\begin{tabular}{l|c|c}
\hline \multicolumn{1}{c|}{ Sistema } & Tempo parado em Horas & $\%$ \\
\hline Elétrico & 13 & 12,5 \\
\hline Ar condicionado & 13 & 12,5 \\
\hline Lubrificação & 27 & 25,8 \\
\hline Motor & 11 & 10,5 \\
\hline Transmissão & 9 & 8,6 \\
\hline Arrefecimento & 10 & 9,6 \\
\hline Freio & 4 & 3,8 \\
\hline Estrutural & 7 & 6,7 \\
\hline Hidráulico & 10,5 & 10,0 \\
\hline \multicolumn{1}{c|}{ Total } & $\mathbf{1 0 4 , 5}$ & $\mathbf{1 0 0}$ \\
\hline
\end{tabular}

Por meio dos dados da Tabela 2, a pesquisa identificou que o principal motivo de parada da carregadeira é para realizar lubrificação, e representa $25,8 \%$ do tempo 
total das paradas. Verifica-se pelos dados da Tabela 2, que a pá carregadeira parou por 27 horas para ser lubrificada.

Tem-se ainda que a falta de lubrificação na carregadeira possa vir a ocasionar outros problemas, como no sistema estrutural do equipamento, que aparece na Tabela 2 com 7\% dos motivos das horas de paradas. Isso devido à falta de manutenção poder proporcionar folgas na caçamba. Assim, pode-se dizer que, em relação aos problemas ocasionados pela lubrificação, o número de horas paradas da carregadeira podem atingir 34 horas, ou seja, $32,8 \%$.

A partir desses dados, chegou-se a conclusão que deveria haver uma melhoria na lubrificação da carregadeira e o tempo de horas paradas deveria ser reduzido. Outro fator relevante são os pontos de lubrificação.

Assim, foi necessário conhecer os pontos de lubrificação da carregadeira, que são 32, conforme podem ser verificados na Tabela 3.

Tabela 3 - Pontos de lubrificação da carregadeira.

\begin{tabular}{c|l|c}
\hline \multirow{2}{*}{ Numeração } & \multicolumn{1}{c}{ Local } & $\begin{array}{c}\text { Quantidade } \\
\text { pontos de lubrificação }\end{array}$ \\
\hline 1 & Base do H & 2 \\
\hline 2 & Base do cilindro de inclinação & 2 \\
\hline 3 & Haste do cilindro de inclinação & 2 \\
\hline 4 & Suporte do link & 2 \\
\hline 5 & Ligação do bracinho com a caçamba & 2 \\
\hline 6 & Ligação do bracinho com o link & 2 \\
\hline 7 & Ligação do H com a caçamba & 2 \\
\hline 8 & Haste do cilindro de elevação & 2 \\
\hline 9 & Base do cilindro de elevação & 2 \\
\hline 10 & Articulação inferior & 2 \\
\hline 11 & Haste do cilindro de direção & 2 \\
\hline 12 & Base do cilindro de direção & 2 \\
\hline 13 & Articulação superior & 1 \\
\hline 14 & Rolamento do trem de força & 1 \\
\hline 15 & Cardam & 1 \\
\hline 16 & Balança dianteira & 2 \\
\hline 17 & Balança traseira & $\mathbf{3 2}$ \\
\hline 18 & Eixo de direção & \\
\hline & Total & \\
\hline
\end{tabular}

É importante destacar que cada um dos pontos de lubrificação do equipamento necessita de uma quantidade de graxa adequada para o bom funcionamento. Pouca graxa ocasiona rangidos devido ao atrito entres partes metálicas, e a quantidade de graxa em excesso pode gerar superaquecimento.

As carregadeiras necessitam de intervenções para a lubrificação das articulações a cada 50 horas que operarem, ou caso aconteça alguma parada não programada derivada da falta de lubrificação. Outro fator a enfatizar é a abrasividade da área 
onde o equipamento atua, isso faz com que o equipamento tenha paradas não programadas consecutivas devido a problemas relacionados com lubrificação.

Ao observar as atividades, foram encontradas soluções diversas para sanar os problemas com o equipamento, como:

- Realizar revisões periódicas;

- Diminuir o tempo da lubrificação manual;

- Modificar sistema de lubrificação manual para lubrificação automática.

Ao realizar uma análise das possíveis soluções apresentadas, foi concluído pelos gestores e equipe de manutenção que realizar revisões periódicas na carregadeira era inviável para a empresa, pois já são realizadas no equipamento as revisões de acordo com o que o fabricante exige para o equipamento.

Outra solução analisada foi diminuir o tempo da lubrificação manual. Conforme análise dos gestores e equipe de manutenção era inviável, pois aumentaria o custo com mão de obra e insumos, além de correr o risco de contaminar o solo com o excesso de lubrificante devido a erros por motivo de agilidade.

Por último, a melhor proposta foi modificar o sistema de lubrificação manual para lubrificação automática. Verificou-se que por mais que houvesse um custo inicial, a lubrificação era viável, uma vez que, os ganhos são maiores. Em verdade, os custos seriam diluídos com a diminuição de custos com mão de obra e redução do tempo de paradas da carregadeira para lubrificação. Isso implica em um aumento na disponibilidade física do equipamento e a empresa ganharia com o aumento da produtividade. No entanto, também foi identificado que por mais que o sistema de lubrificação automático fosse implementado teria que realizar a lubrificação manual em três pontos da carregadeira.

Assim, os gestores aprovaram a medidas e um plano de ação para a execução da implementação da lubrificação automática na carregadeira foi desenvolvido. $O$ Quadro 1 apresenta as ações e o prazo das atividades que foram realizadas para a implementação do sistema de lubrificação automática na carregadeira.

Quadro 1 - Ações para implementar a lubrificação automática na carregadeira.

\begin{tabular}{l|c}
\hline \multicolumn{1}{c|}{ Ações } & Prazo \\
\hline Solicitar aprovação da engenharia & $21 / 05 / 2016$ \\
\hline Providenciar materiais necessários & $30 / 05 / 2016$ \\
\hline Instalar dispositivo & $28 / 06 / 2016$ \\
\hline
\end{tabular}

A equipe de manutenção ficou responsável por verificar os modelos de lubrificação automáticos existentes no mercado. E a escolha levou em consideração o equipamento, as variedades disponíveis no mercado, os custos com a instalação e manutenção do sistema. Após estas análises, a equipe, juntamente com os gestores, decidiu que o viável seria que o modelo QuickLub da fabricante Lincoln. A compra do kit de instalação do sistema de lubrificação QuickLub teve um custo de $\mathrm{R} \$ 14.475,40$ por equipamento.

Para a instalação do sistema de lubrificação automática na carregadeira, foi realizado um planejamento em que as instalações foram realizadas no momento que ocorreram as manutenções preventivas do equipamento. Isso reduziu o tempo de horas paradas do equipamento.

Para a instalação do sistema foi necessário realizar um plano de ação em conjunto com os gestores e mecânicos para que toda a modificação no sistema de lubrificação ocorresse de forma planejada. O Quadro 2 apresenta as ações e os 
prazos que foram executados para implementar o sistema de lubrificação automática.

Quadro 2 - Ações e prazos para a implementação do sistema de lubrificação na carregadeira.

\begin{tabular}{l|c}
\hline \multicolumn{1}{c|}{ Ações } & Prazo \\
\hline Efetuar limpeza nos pontos de lubrificação & $24 / 06 / 2016$ \\
\hline Remover graxeiros do equipamento & $24 / 06 / 2016$ \\
\hline Instalar reservatório & $24 / 06 / 2016$ \\
\hline Instalar linha principal de lubrificação & $26 / 06 / 2016$ \\
\hline Instalar linha secundária de lubrificação & $27 / 06 / 2016$ \\
\hline Realizar testes e ajustes do sistema de lubrificação & $28 / 06 / 2016$ \\
\hline
\end{tabular}

Após implementação da manutenção automática na carregadeira, foi realizado um controle das horas paradas não programadas desse equipamento. A Tabela 4 apresenta os valores no período de 01/07/2016 a 31/10/2016.

Tabela 4 - Paradas não programadas da carregadeira com o sistema de lubrificação automática período de 01/7/2016 a 31/10/2016.

\begin{tabular}{l|c}
\hline \multicolumn{1}{c|}{ Motivo } & Horas paradas \\
\hline Buzina não funciona & 4 \\
\hline Ar condicionado não está resfriando & 4 \\
\hline Vazamento hidráulico & 4 \\
\hline Sinal Sonoro de Ré não funciona & 6 \\
\hline Pino de lubrificação obstruído & 3,5 \\
\hline Freio de estacionamento não funciona & 2 \\
\hline Vazamento hidráulico & 2,5 \\
\hline Baixo rendimento & 11 \\
\hline Farol queimado & 1 \\
\hline Ar condicionado não está resfriando & 6 \\
\hline \multicolumn{1}{c}{ Total } & $\mathbf{4 4}$ \\
\hline
\end{tabular}

Conforme se observa na Tabela 4, a pesquisa identificou que em quatro meses, mesmo número de meses analisados quando a carregadeira não tinha o sistema de lubrificação automática, o valor das horas paradas não programadas da carregadeira atingiu 44 horas. Assim, pode-se calcular que o número de horas paradas com o sistema de lubrificação automático reduziu $57,9 \%$ comparado com os valores apresentados anteriormente.

Em seguida, os valores de horas paradas não programadas foram calculados conforme sistema da carregadeira, conforme se pode verificar na Tabela 5.

Tabela 5 - Horas paradas da carregadeira para manutenção conforme sistema.

\begin{tabular}{l|c|c|}
\hline \multicolumn{1}{c|}{ Sistema } & Tempo parado em Horas & $\%$ \\
\hline Elétrico & 11 & 25 \\
\hline Ar condicionado & 10 & 23 \\
\hline
\end{tabular}




\begin{tabular}{l|c|c}
\hline \multicolumn{1}{c|}{ Sistema } & Tempo parado em Horas & $\%$ \\
\hline Lubrificação & 3,5 & 8 \\
\hline Motor & 11 & 25 \\
\hline Freio & 2 & 4,5 \\
\hline Hidráulico & 6,5 & 14,5 \\
\hline \multicolumn{1}{c|}{ Total } & $\mathbf{4 4}$ & $\mathbf{1 0 0}$ \\
\hline
\end{tabular}

$\mathrm{Na}$ Tabela 5, observa-se que o número de horas paradas não programadas para lubrificação atingiu um valor de 3,5 horas, o que representa $8 \%$ das horas totais. Assim, pode-se afirmar que houve uma redução no tempo de horas paradas não programadas da carregadeira, o que aumentou a disponibilidade física do equipamento. Em relação aos dados apresentados anteriormente à implementação do sistema de lubrificação automática, a redução foi de $87 \%$ nas paradas decorrentes de problemas ligados ao sistema de lubrificação.

Um aspecto importante, é que além de manter os pinos, rolamentos e mancais lubrificados, a modificação do sistema de lubrificação automática trouxe autonomia ao equipamento sobre à lubrificação dos pinos e mancais, pois foi instalado um reservatório de 8 litros para armazenamento de graxa. O equipamento deixou de serem lubrificadas a cada 50 horas manualmente e passou a ser verificada a sua lubrificação a cada 250 horas, sendo que a lubrificação dos pinos e buchas é conciliada com a revisão de lubrificação.

Após a implementação do sistema de lubrificação automática na carregadeira, foi realizado um acompanhamento da atividade na área e ficou constatado que houve um ganho no tempo para lubrificar pinos, mancais e rolamentos em $33,4 \%$, redução de em média 1,5 horas para 1,0 hora.

Em sequência, para identificar possíveis ganhos intangíveis foi realizado um questionário com os executantes da tarefa.

A primeira questão buscou identificar se a melhoria trouxe benefícios ergonômicos na realização das atividades de lubrificação manual. Foi identificado que $100 \%$ dos funcionários sentiram melhorias ergonômicas no processo, uma vez que, um dos motivos é que anteriormente era necessário lubrificar 32 pontos, mas com o sistema de lubrificação automática diminuiu consideravelmente a quantidade de pontos de lubrificação para três. O Quadro 3 apresenta esses pontos.

\begin{tabular}{c|l|c}
\hline \multicolumn{2}{c}{ Quadro 3 - Pontos de lubrificação manual na carregadeira. } \\
\hline \multirow{2}{*}{ Numeração } & \multicolumn{1}{|c}{ Local } & $\begin{array}{c}\text { Quantidade de pontos de } \\
\text { lubrificação }\end{array}$ \\
\hline 1 & Reservatório de graxa & 1 \\
\hline 2 & Cardam & 1 \\
\hline 3 & Eixo de direção & 1 \\
\hline
\end{tabular}

A segunda questão abordou se os funcionários e mecânicos aprovam o sistema de lubrificação automática. Por meio da resposta dos entrevistados, a pesquisa identificou que todos estavam muito satisfeitos com o novo sistema.

Sem levar como fator de geração de lucro a maior disponibilidade do equipamento e o aumento da vida útil dos pinos e buchas, o simples fato de diminuir o tempo do processo de lubrificação do equipamento, disponibilizando a mão de obra para 
outras atividades e otimizando o processo de manutenção em geral, que por fim obteve-se lucro.

Notou-se com os resultados da análise dos dados que a modificação do sistema de lubrificação automática proporcionou melhoria no processo de lubrificação, diminuiu custos, otimizou a mão de obra e elevou a moral da equipe, com uma melhor condição de trabalho e atendendo a necessidade dos executantes.

\section{CONCLUSÕES}

Como a cada dia as indústrias crescem, produzem, vendem e exigem mais dos seus equipamentos se faz necessário também realizar uma perfeita manutenção. $\mathrm{Na}$ empresa onde ocorreu este estudo, viu-se a necessidade de melhorias em relação à manutenção de lubrificação na pá carregadeira. A gestão da manutenção aparece neste cenário como uma oportunidade de otimização de recursos, que envolve redução de custos, tempo de indisponibilidade dos equipamentos, paradas não programadas e investimentos em equipamentos novos.

Manter os equipamentos na maior parte do tempo disponíveis se torna um compromisso extremamente necessário para a estratégia de crescimento e desenvolvimento empresarial.

Com esse estudo pode-se concluir, por meio da implementação do sistema de lubrificação automática na pá carregadeira da mineradora, que melhorias no processo de manutenção são essenciais para que empresas se tornem competitivas no mercado. Foram observados diversos pontos positivos da implantação do sistema de lubrificação automática:

- Redução de custos com relação a insumos e mão de obra;

- Maior disponibilidade do equipamento;

- Diminuição de riscos de acidentes;

- Aumento da produtividade.

O estudo demonstra que contribui para que outras empresas e empresários vejam que o dinheiro gasto com a instalação do sistema de lubrificação automática não é um custo e sim um investimento e o maior beneficiado será o mesmo.

\section{REFERÊNCIAS}

1 COLLINS, J. A. Projeto mecânico de elementos de máquinas: uma perspectiva de prevenção da falha. Tradução de Pedro Manuel Calas Lopes Pacheco et al. Rio de Janeiro: LTC, 2006.

2 IPIRANGA, Companhia Brasileira de Petróleo. Lubrificantes industriais. Disponível em < http://www.ipiranga.com.br >. Acesso em 15 de outubro de 2016.

3 PIZZANI, Química Industrial. Molykote Lubrificantes. Disponível em < http://www.graxalubrificante.com.br >. Acesso em 26 de setembro de 2016.

4 SANTOS, Francine Silva Garcia dos. Graxas lubrificantes e lubrificantes especiais. Disponível em < https://amigonerd.net/exatas/engenharia/graxaslubrificantes-e-lubrificantes-especiais>. Acesso em 24 de outubro de 2016.

5 FRANZEN, Grupo. Catalogo Lincoln. Disponível em < http://www.franzen.com.br >. Acesso em 26 de setembro de 2016.

6 EXIMPORT, Lubesystems. Princípios da Lubrificação. 2005. Disponível em < http://www.eximport.com.br>. Acesso em 04 de setembro de 2016. 
7 Carmassa Brasil - CMB. Lubrificação Industrial Centralizada. Disponível em <http://www.lubrificacaocentralizada.com>. Acesso em 14 de outubro de 2016.

8 SKF, Grupo. O poder da lubrificação. Disponível em < http://www.skf.com >. Acesso em 22 de outubro de 2016.

9 M\&T, Revista Manutenção e Tecnologia. Forças que atuam sobre a carregadeira. 2010. Disponível em:<http://www.revistamt.com.br>. Acesso em: $31 / 10 / 2016$

10 Umarizal Terraplanagem e Locação - ULT. Pá Carregadeiras. Disponível em < http://www.umarizal.com/equipamentos/pa-carregadeiras/>. Acesso em 31 de outubro de 2016. 